Après avoir démontré - ce qui ne comporte aucune difficultí les théorèmes de la théorie des fonctions caractéristiques, analogues à ceux de M. Glivenko ${ }^{10}$ ) et M. Oramér ${ }^{11}$ ) pour les lois de probabilité de deux variables, on peut remplacer la convergence uniforme de la suite de fonctions caractéristiques par des conditions plus faibles.

10) V. Glivenko, Sul teorema limite della teoria delle funzioni caratteristiche, Giorn. Istit. Attuari 7 (1936), p. 160-167.

11) H. Cramér, Random variables and Probability Distributions, Cambridge 1937, p. 29-31 et 121.

\section{Sur le prolongement des transformations en surfaces sphériques.}

Par

\section{Samuel Eilenberg (Warszawa).}

Etant donnés dans une variété $n$-dimensionnelle a deux ensembles fermés et disjoints $X$ et $Y$, on peut étudier leur situation mutuelle dans 20 soit par la méthode d'homologie ${ }^{1}$ ), soit, dans quelques questions particulières, par la considération du groupe fondamental de $2 e-X-Y^{2}$ ).

La troisième méthode, très peu développée, est celle de mettre en jeu aussi les transformations continues $f$ de $X$ en sous-ensembles d'un espace $\mathfrak{\Xi}$ convenablement choisi et d'envisager ces transformations au point de vue de l'existence de leurs prolongements $f($ ae $-Y) C \cong$.

O'est ainsi que M. Borsuk a examiné récemment ${ }^{3}$ ) le cas où $\mathfrak{D}$ est une surface sphérique $n$-dimensionnelle $S^{n}$ et $X=\mathfrak{F}$, en aboutissant à des conditions nécessaires de nature homologique (et qui sont aussi suffisantes dans quelques cas spéciaux), pour que toute transformation $f(X) \subset X$ admette un prolongement $\left.f\left(S^{n}-\bar{X}\right) \subset X^{4}\right)$.

Dans la suite, nous allons fixer $\cong$ : ce sera toujours la surface sphérique $m$-dimensionelle $S^{m}(m>0)$. Nous aboutirons à des conditions pour que toute transformation $f(X) \subset S^{m}$ admette un prolongement $f(\mathfrak{C}-Y) \subset S^{m}$. Ces conditions seront également homologiques, mais cette fois elles seront suffisantes (sans être généralement nécessaires).

1) Yoir p.ex. S. Lefschetz, Topology, New-York 1930, p. 142.

2) K. Reidemeister, Knotentheorie, Berlin, Springer 1932.

s) K. Bors uk, Fund. Math. 29 (1937), p. 191-205.

4) c. àd. pour que $X$ soit un rétracte de $S^{n}-Y$. 
La partie I contient l'exposé de la méthode et l'énoncé du lemme principal, dont la démonstration sera remise à la partie III. La partie II est consacrée à quelques appliquations du lemme principal; les parties II et III peuvent être lues indépendemment l'une de l'antre.

1. Préliminaires, de désignera un espace métrique compact, donné dans une décomposition fixe

$$
\mathfrak{Q}=X+Q+Y \quad \text { où } \quad X \cdot X=X \cdot Q=X \cdot Q=0,
$$

$X$ et $Y$ désignant des ensembles fermés et $Q$ un polyèdre simplicial infini ${ }^{5}$ ).

Tous les simplexes de $Q$ seront supposés orientés. Un simplexe $k$-dimensionnel (orienté) sera désigné par $a_{l}^{k}$ et l'ensemble fermé qui lui correspond par $\left|a_{t}^{k}\right|$. La chaine $(k-1)$-dimensionnelle qui est la trontière combinatoire de $a_{i}^{k}$ sera désignee par $c a_{l}^{h}$.

2. Cycles infinis mod $\boldsymbol{Y}^{6}$ ). Soit $(\mathcal{G}$ un groupe abelien. Une fonction quelconque qui fait correspondre à tout simplexe $k$-dimensionnel $a_{i}^{k}$ de,$Q$ un élément $\alpha_{i} \in \mathcal{G}$ sera désignée par

$$
\Lambda^{k}=\sum \alpha_{i} a_{i}^{k}
$$

et appellée chaîne k-dimensionnelle infinie à coefficients de $\mathcal{G}$.

Posons

$$
\left|\Lambda^{k}\right|=\sum_{i}\left|a_{i}^{k}\right|
$$

où la sommation s'étend à tous les indices $i$ pour lesquels $\alpha_{l} \neq 0$. On dira que $\Lambda^{k}$ est une chaîne $\bmod Y$, lorsque

$$
\left.\overline{\left|\Lambda^{k}\right| \cdot X=0}{ }^{7}\right) \text {. }
$$

5) Un polyèdre infini est un ensemble $Q$ qui admet une décomposition sim. pliciale, c. àd. une décomposition de la forme $Q=\sum_{i=1}^{\infty} \Delta_{i}$ où $\Delta_{t}$ sont des simplexes géometriques fermés assujettis aux conditions:

1) $\Delta_{i} \Delta_{j}$ est une face (de dimension $\geqslant-1$ ) de $\Delta_{i}$ et de $\Delta_{j}$

2) aucun point de $Q$ n'est un point d'accumulation d'une suite de points appartenant à de divers termes de la suite $\left\{\Delta_{l}\right\}$.

Ainsi, un polyèdre infini est localement un polyèdre fini. La décomposition simpliciale de $Q$ étant fixée, nous dirons que $Q$ est un polyèdre infini simplioial.

$\left.{ }^{6}\right)$ Cf. S. Lefschetz, l. cit., Chap. VII.

7) $\bar{A}$ désigne la fermeture de $A$ dans a.
Soit

$$
\partial \Lambda^{k}=\sum_{i} a_{i} \partial \alpha_{i}^{k}
$$

Si $\partial \Lambda^{k}=0$, on dira que $\Lambda^{k}$ est un eycle infini. Si, de plus, $\Lambda^{k}$ est une chaîne mod $Y$, on dira que $\Lambda^{k}$ est une cycle infini mod $Y$ (à coetficients du groupe $\mathcal{S}$ ).

Un cycle infini $k$-dimensionnel $\gamma^{k} \bmod Y$ (coefficient de $\mathcal{G}$ ) sera dit homologue $\dot{a}$ zéro $\bmod Y$, en symboles:

$$
\gamma^{k} \sim 0 \bmod Y,
$$

lorsqu'il existe une chaine $(k+1)$-dimensionnelle $\Lambda^{k+1} \bmod Y$ (à coefficients de $\mathcal{G}$ ) telle que

$$
\gamma^{k}=\partial 1^{k+1} .
$$

3. Cellules duales. Admettons maintenant que $Q=Q^{n}$ est une variété orientable à $n$-dimensions $\left.{ }^{8}\right)(n>0)$. Nous considérons la composition de $Q^{n}$, duale à celle en $a_{i}^{k}$. Les cellules de cette décomposition seront dites cellules duales tout court. La cellule duale à $a_{i}^{k}$ sera désignée par $b_{i}^{n-k}$ et orientée de façon à avoir

$$
\left.\chi\left(a_{i}^{k} ; b_{i}^{n-k}\right)=1 \quad{ }^{9}\right) \text {. }
$$

L'ensemble fermé qui correspond à $b_{i}^{n-k}$ sera désigné par $\left|b_{i}^{n-k}\right|$ et la frontière combinatoire de $b_{i}^{n-k}$ par $s_{i}^{n-k-1}$ :

$$
s^{n-k-1}=\partial b_{i}^{n-k} .
$$

Le polyèdre $\left|s_{i}^{n-k-1}\right|$ est homéomorphe à la surface sphérique $(n-7-1)$-dimensionnelle $S^{n-k-1}$, que nous supposons orientée d'une manière fixe. D'autre part, $\left|s_{i}^{n-k-1}\right|$ sera supposé orienté par le cycle (à coefficients entiers) $s_{i}^{n-k-1}$. Ceci fait, nous pourons fixer une transformation continue

$$
\varphi_{i}^{n-k-1}\left(S^{n-k-1}\right) \subset\left|s_{i}^{n-k-1}\right|
$$

parmi celles qui ont le degré 1.

8) Pour tout ce qui concerne la définition des variétés et des cellules duales, voir S. Lefschetz, Topology, Ch. III, \$2.

${ }^{9}$ L'opérateur $\chi$ désigne le coeficient d'intersection. 
4. Les groupes $\left(m^{i}\right)$. Considérons, pour un $m$ positif, les transformations continues $g$ de $S^{l}$ en sous-ensembles de $s^{y^{m}}$; en symboles:

$$
g\left(S^{i}\right) C S^{m} \quad \cdot \quad(m>0) .
$$

La classe des transformations homotopes à g sera dlésignée par $[g]$. Etant donnés deux classes $\left[g_{1}\right]$ et $\left[g_{2}\right]$, on cléfinit la classe $\left[g_{1}\right]+\left[g_{2}\right]$ comme il suit:

Soit $S^{i}=S_{-}^{i}+S_{+}^{i}$ la décomposition de $S^{l}$ en deux hemisphères et soit $q \in S^{m}$. Il existe alors deux transformations $g_{1}^{\prime} \in\left[g_{1}\right]$ et $g_{2}^{\prime} \in\left[g_{2}\right]$ telles que:

$$
\left.\begin{array}{lll}
\text { pour tout } & x \in S_{-}^{i} & g_{1}^{\prime}(x) \\
\text { pour tout } & x \in S_{+}^{l} & g_{2}^{\prime}(x)
\end{array}\right\}=q .
$$

Posons:

$$
g(x)=\left\{\begin{array}{lll}
g_{1}^{\prime}(x) & \text { pour tout } x \in S_{+}^{l} \\
g_{1}^{\prime}(x) & \text { pour tout } x \in S_{-}^{l}
\end{array}\right.
$$

et

$$
[g]=\left[g_{1}\right]+\left[g_{2}\right] .
$$

On peut démontrer que cette addition est univoque et qu'elle conduit à un groupe abelien au plus dénombrable; nous les désignerons par $\left.\left(m^{i}\right)^{10}\right)$. L'élément 0 de ce groupe correspond à la classe des transformations inessentielles.

Le groupe $\left(m^{i}\right)$ à été calculé dans les cas suivants:

$1^{0}\left(m^{i}\right)$ pour $i<m$. Toute transformation. $g\left(S^{l}\right) \subset S^{m}$ est alors inessentielle et le groupe $\left(m^{t}\right)$ se réduit à l'élément 0 .

$2^{0}\left(m^{m}\right)$; la classe d'une transformation $g\left(S^{m}\right) \subset S^{m}$ est définio biunivoquement par le degré de $\left.g^{11}\right)$; de-même, l'addition de classes correspond à celle de degrés. Le groupe $\left(m^{m}\right)$ est donc cyclique infini ${ }^{12}$ ):

$\left.{ }^{10}\right)$ Le groupe $\left(m^{i}\right)$ n'est rien d'autre que le $i$-ième groupe d'homotopie $\pi_{i}\left(S^{m}\right)$ de $S^{m}$ introduit par M.W. Hurewicz (Proceed. Akad. Amsterdam 38 (1935), p. 113). La définition du texte est celle de M. K. Borsuk (C. R. Paris 202 (1936), p. 1400-3). La notation $\left(m^{l}\right)$ est due d M. H.Freudenthal (Comp. Math. 5 (1937), p. 299).

11) P. Alexandroff et H. Hopf, Topologie I, Berlin, Springer 1935, p. 501-505; H. Whitney, Duke Math. Journ. 3 (1937), p. 46-50.

12) W. Hurewicz, l. cit., p. 114.
$3^{0} \quad\left(m^{m+1}\right)$ pour $m \geqslant 3$ est cyclique d'ordre $\left.2^{13}\right)$.

$4^{0} \quad\left(m^{m+2}\right)$ pour $m \geqslant 3$ se réduit à l'élément $\left.0^{14}\right)$.

$5^{0} \quad\left(1^{i}\right)$ pour $i \geqslant 2$ se réduit à l'élément $\left.0^{15}\right)$.

$6^{0} \quad\left(2^{i}\right)$ et $\left(3^{i}\right)$ sont toujours isomorphes $\left.{ }^{16}\right)$.

On déduit ${ }^{17}$ ) facilement de $2^{0}$ que

(4.1) $\varphi\left(S^{i}\right) \subset S^{i}$ étant une transformation de degré $\eta$, on a pour toute transformation continue $g\left(S^{i}\right) \subset S^{m}$

$$
[g \varphi]=\eta[g] .
$$

5. Définition de $\gamma^{k}(f)$. Soit $P^{k}$ un sous-polyèdre $k$-dimensionnel de $Q^{n}{ }^{18}$ ), tel que $\bar{P}^{k} \cdot X=0$. Considérons une transformation continue

$$
f\left(X+Q^{n}-P^{k}\right) \subset S^{m}
$$

Pour tout $a_{i}^{k}$ de $Q^{n}$, on a donc $\left|s_{i}^{n-k-1}\right| \subset Q^{n}-P^{k}$, et la transformation

$$
f p_{i}^{n-k-1}\left(S^{n-k-1}\right) \subset S^{m}
$$

est bien définie. Posons

$$
\alpha_{i}^{n}(f)=\left[f p_{i}^{n-k-1}\right] \epsilon\left(m^{n-k-1}\right) .
$$

Toute transformation continue $\varphi\left(S^{n-k-1}\right) \subset\left|s_{i}^{n-k-1}\right|$ de degré 1 étant homotope à $\varphi_{i}^{n-k-1}$, on a $[f p]=\left[f \varphi_{i}^{n-k-1}\right]$. L'élément $\alpha_{i}(f)$ est done défini univoquement par $a_{i}^{k}$ et par la transformation (5.1).

Posons

$$
\gamma^{k}(f)=\sum_{i} a_{i}(f) a_{i}^{k}
$$

où la sommation s'étend à tous les $a_{i}^{k}$ de $Q^{n}$.

13) L. Pontrjagin, Congrès International de Mathématiciens, Oslo 1936, Tome II, p. 140; H. Freudenthal, l. cit., p. 301.

14) L. Pontrjagin, l. cit.

15) Alexandroff-Hopf, l. cit., p. 515

18) W. Hurewicz, 1. cit., p. 119.

17) H. Freudenthal, 1. cit., p. 303.

18) Sous-polyedre de $Q^{n}$ veut dire: somme (d'un nombre fini ou non) de simplexes (fermés) de la subdivision simpliciale fixée de $Q^{n}$. Le terme n-dimen sionnel est employé ici toujours dans le sens d'au plus n-dimensionnel. 
Si $\left|a_{i}^{k}\right| n o n \subset P^{k}$, on a $\left|b_{i}^{n-k}\right| \subset Q^{n}-P^{k}$. La transformation $f$ est done inessentielle sur $\left|s_{i}^{n-k-1}\right|$ et par conséquent la transformation (5.2) est inessentielle, cà d. $\alpha_{i}(f)=0$. Il en résulte que

$$
\left|\gamma^{k}(f)\right| \subset P^{k}
$$

6. Lemme principal. $\gamma^{k}(f)$ est un oyole infini le-dimensionnel $\bmod Y$ à coefficients de $\left(m^{n-k-1}\right)$.

La relation $\gamma^{k}(f) \sim 0$ mod $Y$ implique l'existence: d'un souspolyèdre $(k-1)$-dimensionnel $P^{k-1}$ de $Q^{n}$ tel que $\overline{P^{k-1}} \cdot X=0$, et d'une transformation continue

$$
f^{*}\left(X+Q^{n}-P^{k-1}\right) \subset S^{m}
$$

qui coïncide avee $f$ sur $X$.

Ge lemme sera démontré dans la partie III.

7. Parallélisme de $f$ et de $\gamma^{k}(f)$, On a en vertiu de (5.4) et de (3.1)

$$
\chi\left[\gamma^{k}(f) ; b_{i}^{n-k}\right]=\chi\left[\alpha_{l}(f) a_{i}^{k} ; b_{i}^{n-k}\right]=\alpha_{i}(f)
$$

donc d'après $(3.2)$

$$
\left.v\left[\gamma^{k}(f) ; s_{i}^{n-k-1}\right]=\alpha_{i}(f) .{ }^{19}\right)
$$

Désignons par $\hat{Q}^{n}$ la subdivision barycentrique de $Q^{n}$. Soit $\hat{\Lambda}^{n-h}$ une chaîne finie $(n-k)$-dimensionnelle de $\hat{Q}^{n}$ à coefficients d'un groupe abelien $\mathcal{G}$, telle que $\left|\partial \hat{\Lambda}^{n-k}\right| C \hat{Q}^{n}-P^{k}$. On trouve alors facilement une suite finie $\beta_{1}, \beta_{2}, \ldots, \beta_{r} \in \mathcal{G}$ telle que

$$
\partial \hat{\Lambda}^{n-k} \sim \sum_{i=1}^{r} \beta_{i} s_{i}^{n-k-1} \quad \text { dans } \quad \hat{Q}^{n}-P^{k},
$$

ce qui implique en vertu de $(7: 2)$ que

$$
\chi\left[\gamma^{k}(f) ; \hat{\Lambda}^{n-k}\right]=\sum_{i=1}^{r} \beta_{i} \alpha_{t}(f) .
$$

Etant donné un cycle fini $m$-dimensionnel $\hat{\gamma}^{m}$ de $\hat{Q}^{n}-P^{k} \grave{\text { à coef- }}$ ficients de $\mathcal{G}$, on désignera par $\mathrm{g}\left(f ; \hat{\gamma}^{m}\right)$ le degré de la transformation $f$ sur le cycle $\hat{\gamma}^{m}{ }^{20}$ ).

$\left.{ }^{19}\right)$ L'opérateur $v$ désigne le coefficient d'enlacement.

$\left.{ }^{20}\right)$ Le groupe $B^{m}\left(S^{m}\right)$ coef. $\subseteq$ étant isomorphe a $g$, on définit $g\left(f, \hat{\gamma}^{m}\right)$ comme l'élément de $B^{m}\left(S^{m}\right)$ ooef. $\mathcal{G}$ déterminé par lo cyole $f\left(\hat{\gamma}^{m}\right)$; of. p. ex. Alexandrof. Hopf, 1. cit., p. 491 .
Admetitons que $n=m+k+1$. On a alors $n-k-1=m$ et l'élement $\mathrm{g}\left(f ; \partial \hat{\Lambda}^{n-k}\right) \in \mathcal{S}$ est bien déterminé. L'élément $a_{i}(f)$ étant, comme on le déduit de sa délinition, égal à $g\left(f ; s_{i}^{n-k-1}\right)$, on tire de (7.3) et $(7.4)$

$$
\chi\left[\gamma^{k}(f) ; \hat{\Lambda}^{n-k}\right]=g\left(f ; \partial \hat{\Lambda}^{n-k}\right) .
$$

Admettons, de plus, que $\mathfrak{e}=S^{n}=Q^{n}$ et $X=Y=0$. Pour tout cycle $\hat{\gamma}^{m}$ de $\hat{Q}^{n}-P^{k}$, on a alors $\hat{\gamma}^{m} \sim 0$ dans $\hat{Q}^{n}$, ce qui implique en vertu de (7.5) que

$$
v\left[\gamma^{k}(f) ; \hat{\gamma}^{m}\right]=\mathrm{g}\left(g ; \hat{\gamma}^{m}\right) .
$$

On voit done que $f$ et $\gamma^{k}(f)$ sont parallèles dans le sens introduit par M. Borsuk et moi ${ }^{21}$ ) pour $m=1$.

8. Le cas de $Q^{n}$ non orientable. Même dans ce cas, ]a définition de $\gamma^{k}(f)$ et le lemme principal restent valables, si l'on suppose que chaque élément du groupe $\left(m^{n-k-1}\right)$ est d'ordre 2. On a alors $\alpha=-\alpha$ pour tout $\alpha \epsilon\left(m^{n-k-1}\right)$ et la définition (5.3) de $\alpha_{i}(f)$ reste univoque, malgré que $b_{i}^{n-k}$ soit non orientée et que $s_{i}^{n-k-1}$ soit un cycle $\bmod 2$.

II

9. Cycles convergents $\bmod { }^{22}$ ) Soit $\mathscr{Y}$ un espace métrique et $Y \subset \mathscr{V}$ un ensemble fermé.

Le complexe algébrique $k$-dimensionnel $e^{k}$ (à coefficients appartenant à un groupe abelien $\mathcal{G}$ ) est dit $\varepsilon$-complexe ou $\varepsilon$-châ̂ne de $A \subset \mathscr{V}$, lorsque tous ses sommets appartiennent à $A$ (en symboles: $c^{k}(A)$ et que la distance entre deux sommets, d'un simplexe de $c^{k}$ reste inférieure à $\varepsilon$.

$z^{k}$ est dit $\varepsilon$-oyele $k$-dimensionnel $\bmod Y$ de $A$, lorsque $z^{k}$ est une $\varepsilon$-chaîne $k$-dimensionnelle de $A$ et $\hat{c} z^{k} \subset Y$.

Le $\varepsilon$-cycle $z^{k} \bmod Y$ est dit $\varepsilon$-homologue à zéro $\bmod Y$ dans $A$, en symboles:

$$
z^{k} \approx 0 \bmod Y \text { dans } A,
$$

lorsqu'il existe une $\varepsilon$-chaîne $(k+1)$-dimensionnelle $c^{k+1} C A$ telle que $z^{k}-\partial 0^{k+1} \subset Y$.

21) K. Borsuk et S. Eilenberg, Fund. Math. 26 (1936), p. 210.

${ }^{22}$ ) Cf. L. Vietoris, Math. Ann. 97 (1927), p. 458; S. Lefschetz, Ann. of Math. 29 (1928), p. 232-254: P. Alexandroff. Math. Ann. 106 (1932), p. 191. 
Une suite

$$
Z^{h}=\left(z_{1}^{h}, z_{2}^{h}, \ldots\right)
$$

est dite vrai cycle k-dimensionnel mod $I$ de $A$, lorsqu'il existe un ensemble compact (en soi) $A_{1} C A$ et une suite de nombres positifs $\varepsilon_{i} \rightarrow 0$, tels que $z_{i}^{k}$ est un $\varepsilon_{i}$-cycle $k$-dimensionnel $\bmod Y$ de $A_{1}$. symboles:

Un vrai cycle $Z^{k}$ est dit homologue à zéro $\bmod Y$ dans $A$, on.

$$
Z^{k} \sim 0 \bmod X \text { dans } A,
$$

lorsqu'il existe un ensemble compact $A_{1} C A$ et une suite $\varepsilon_{l} \rightarrow 0$, tels que $z_{i}^{k} \tilde{\varepsilon}_{\varepsilon_{i}} 0 \bmod Y$ dans $A_{1}$.

Un vrai cycle $Z^{k} \bmod Y$ de $A$ est dit oonvergent, lorsqu'il existe un ensemble compact $A_{1} \subset A$ et une suite $\varepsilon_{i} \rightarrow 0$, tels que $\approx_{i}^{h}-z_{i+1}^{h} \tilde{n}_{i} 0$ $\bmod X$ dans $A_{1}$.

Le groupe d'homologie qui s'obtient en considérant les oycless k-dimensionnels convergents $Z^{k}$ mod $Y$ de $A$. (les coefficientis dtant pris du groupe $\mathcal{G})$ sera désigné par

$$
B^{k}(A) \bmod Y \text { coef. } \mathcal{S} \text {. }
$$

Dans le cas $Y=0$ le signe „mod $Y$ " sera tout simplement omis.

10. Passage des cycles infinis aux cycles convergents.

Dans les applications qui vont suivre, on admettra toujours que:

$1^{0}$ de est un rétracte absolu de voisinage ${ }^{23}$ ),

$2^{0} \quad X C \mathfrak{C}$ est un ensemble fermé,

$3^{0} \quad Y C \mathfrak{C O}-X$ est un rétracte absolu de voisinage $\left.{ }^{24}\right)$,

$4^{0}$ l'ensemble ouvert $\mathfrak{e}-X-Y$ est donné dans une décomposition simpliciale (infinie) $Q^{n}$ qui est une variété orientable àn $n$ dimensions.

On sait ${ }^{25}$ ) que dans ces conditions

(10.1) l'étude des cycles infinis $\gamma^{k} \bmod X$ de $Q^{n}$ équivaut $\grave{a}$ celle des cycles convergents $Z^{k}$ mod $Y$ de $20-X$.

$\left.{ }^{23}\right)$ K. Borsuk, Fund. Math. 19 (1932), p. 222.

$\left.{ }^{24}\right)$ Au lieu de $1^{0}$ et $3^{0}$, il suffit d'admettre que $2 e$ est wn espace métrique compact et que $Y \subset{ }^{2}-X$ est un ensemble fermé tel que

$(R)$ pour tout entourage $U$ de $Y$, il existe un entourage $V$ de $X$ tel que elaque cyole convergent mod $Y$ de $V$ est homologue à zéro mod $Y$ dans $U$.

${ }^{25}$ ). L'idée de la démonstration est indiquée par M. S. Lefschetz (T'opo. logy, p. 330-334). La démonstration détaillée est assez simple; elle fait usago de la propriété $(R)$ de ${ }^{24}$ ).
Soit $\mathscr{S}$ un groupe abelien discret et an plus dénombrable. $\mathcal{S}^{*}$ désignera le groupe topologique compact, orthogonal ${ }^{26}$ ) à $\mathfrak{S}$. En particulier, $\left(m^{m}\right)$ étant isomorphe au groupe des nombres entiers, $\left(m^{m}\right)^{*}$ l'est à celui des nombres réels réduits mod 1 .

Soient: $Z_{1}^{k}$ un cycle convergent $k$-dimensionnel $\bmod Y$ de $\mathfrak{D e}-X$ à coefficients de $\mathscr{G}$ et $Z_{2}^{n-k}$ un cycle convergent $(n-k)$-dimensionnel $\bmod X$ de $\mathfrak{C}-Y$ à coefficients de $\mathfrak{S}^{*}$. Allors:

(10.2) le coefficient d'intersection $\chi\left(Z_{1}^{k} ; Z_{2}^{n-k}\right)$ dettermine l'orthogonalité des groupes:

$B^{k}(\mathfrak{e}-X) \bmod Y$ coef. $\mathcal{S}, \quad B^{n-k}(\mathfrak{C}-Y) \bmod X$ coef. $\left.\mathcal{S}^{*}{ }^{27}\right)$.

11. Theorème 1. Supposons que

$$
\left.B^{i+1}(\mathfrak{C}-Y) \bmod X \text { coef. }\left(m^{i}\right)^{*}=0^{28}\right)
$$

et soit $f(X) \subset S^{m}$ une transformation continue.

$S^{\prime} i l$ existe un sous-polyèdre $(n-i-1)$-dimensionnel $P^{n-i-1}$ de $Q^{n}$ tel que $\overline{P^{n-i-1}} \cdot X=0$, et si la fonction $f$ admet un protongement

$$
f\left(\mathfrak{e}-Y-P^{n-i-1}\right) \subset S^{m},
$$

alors il existe aussi un sous-polyèdre $(n-i-2)$-dimensionnel $P^{n-i-2}$ de $Q^{n}$ tel que $\overline{P^{n-i-2}} \cdot X=0$ et que $f$ admet un prolongement

$$
f\left(\mathfrak{C}-Y-P^{n-i-2}\right) \subset S^{m} \text {. }
$$

En effet, soit $\gamma^{n-i-1}(f)$ le cycle infini $\bmod Y$ de $Q^{n}$ à coefficients de $\left(m^{i}\right)$, associé à (11.2). Wn vertu de (11.1.) $)_{i},(10.2)$ et (10.1), on a aloris $\gamma^{n-i-1}(f) \sim 0 \bmod Y$ dans $Q^{n}$. L'existence du polyèdre $P^{n-i-2}$ et $d u$ prolongement (11.3) résulte donc du lemme principal.

${ }^{26)}$ L. Pontrjagin, Ann. of Math. 35 (1934), p. 369.

27) C'est un théorème correspondant dans la théorie d'orthogonalité de M. L. Pontrjagin (Ann. of Math. 35 (1934), p. 912), au théorème de dualité de M. S. Lefschetz (Topology, p. 142 et p. 314). Dans la démonstration, on répète partiellement le raisonnement de M. Pontrjagin (c.. aussi Math. Ann. 105 (1931), p. 190) et le raisonnement employé dans la démonstration de (10.1) (cf. renvoi $\left.{ }^{25}\right)$ ). Les démonstrations des propositions (10.1) et (10.2), essentielles pour les théorèmes qui suivent, exigent une étude spéciale dépassant les fins de cet article. ${ }^{28}$ ) c. à d. que le groupe en question se réduit à l'élément 0. 
Theorème $\boldsymbol{I r}^{29}$ ) Pour toute transformation continue $f(X) \subset \mathrm{S}^{m}$, il existe un sous-polyèdre $(n-m-1)$-dimensionnel $P^{n-m-1}$ de $Q^{n}$, tel que $\overline{P^{n-m-1}} \cdot X=0$ et que $f$ admet un prolongement

$$
f\left(\mathfrak{C}-X-P^{n-m-1}\right) \subset \mathcal{S}^{m}
$$

$S^{m}$ étant un rétracte absolu de voisinage, il existie un entourage $U$ de $X$ et un prolongement $\left.f(U) \subset S^{m}{ }^{23}\right)$. Il existe done un sous-polyèdre $n$-dimensionnel $P^{n}$ de $Q^{n}$ tel que $\overline{P^{n}} \cdot X=0$ et un prolongement $f\left(\mathfrak{C}-Y-P^{n}\right) \subset S^{n}$. Désignons par $P^{n-1}$ la somme de tous les simplexes au plus $(n-1)$-dimensionnels de $P^{n}$ et posons $f(x)=q \in S^{m}$ pour tout $x \in P^{n}-P^{n-1}$. On a ainsi $\overline{P^{n-1}} \cdot X=0$ et $f\left(2 e-X-P^{n-1}\right) \subset S^{m}$.

Pour $i=0,1, \ldots, m-1$, on a $\left(m^{\prime}\right)=0$ et la condition (11.1.), est alors automatiquement satisfaite. En appliquant done le th. T successivement à $i=0,1, \ldots, m-1$, on aboutit aे $P^{n-m-1}$ ot au prolongement (1.1.4).

12. Etude du cycle $\gamma^{n-m-1}(f)$. Soit $\left.f(X) \subset S^{m l}{ }^{30}\right)$. A tout cyclo convergent $m$-dimensionnel $Z^{n l}$ de $X$ à coefficients pris de $\left(m^{m}\right)^{*}$ correspond un élément de $\left(m^{m}\right)^{*}$ qui est le degré de $f$ sur $Z^{m}$. Nous le désignerons par $\mathrm{g}\left(f ; Z^{m}\right)$.

En faisant correspondre à tout cycle convergent $(m-1)-1)$-dimensionnel $Z^{m+1} \bmod X$ de $2 e-Y$ à coefficients de $\left(m^{m}\right)^{*}$ l'élément $\mathrm{g}\left(f ; \partial Z^{m+1}\right) \epsilon\left(m^{m}\right)^{*}$, on obtient un caractère $\left.{ }^{26}\right)$ du groupe

$$
B^{m+1}(\mathfrak{e}-Y) \bmod X \text { coef. }\left(m^{m}\right)^{*} \text {. }
$$

La transformation $f$ sera dite algébriquement prolongeable sur de $-Y$, lorsque ce caractère est $0, c$ à $d$. lorsqu'on a toujours $\mathrm{g}\left(f ; \partial Z^{m+1}\right)=0$.

Evidemment, c'est une condition nécessaire pour l'existence d'un prolongement $f(\mathfrak{Q}-Y) C S^{m}$; elle est toujour, satisfaite si $B^{m+1}(\partial e-Y) \bmod X$ coef. $\left(m^{m}\right)^{*}=0$ ou bien $B^{m}(X)$ coef. $\left(m^{m}\right)^{*}=0$.

Le caractère qui vient d'être attaché à $f$ détermine en vertu de (10.2) et (10.1) une classe d'homologie $\bmod Y$ de cycles infinis $(n-m-1)$-dimensionnels $\gamma^{n-m-1} \bmod Y$ de $Q^{n}$ à coefficients de $\left(m^{m}\right)$.

$\left.{ }^{29}\right)$ Ce théorème se laisse établir d'une fuçon tout a fait élémentaire; of. S. Eilen berg, Fund. Math. 26 (1936), p. 280 et K. Borsuk, Fund. Math. 29
(1937), p. 162.
Nous désignerons cetie classe par $\Gamma^{n-m-1}(f)$. Les cycles

$$
\gamma^{n-m-1} \in \Gamma^{n-m-1}(f)
$$

sont caractérisés par l'équation

$$
\chi\left(\gamma^{n-m-1} ; Z^{m+1}\right)=\mathrm{g}\left(f ; \partial Z^{m+1}\right),
$$

qui est satisfaite pour chaque cycle coirvergent $(m+1)$-dimensionnel $Z^{m+1} \bmod X$ de ${ }^{e}-Y$ à coefficients de $\left(m^{m}\right)^{*}$. Il en résulte qu'on a $\Gamma^{n-m-1}(f)=0$ dans le cas où $f$ est algébriquement prolongeable sur $\mathfrak{D}-Y$ et seulement dans ce cas.

Sojent maintenant: $P^{n-m-1}$ le polyèdre donné par le th. II et $\gamma^{n-m-1}(f)$ le cycle qui. correspond au prolongement (11.4). Il résulte de $(7.5)$ que $\gamma^{n-m-1}(f)$ satisfait à (12.1), donc que $\gamma^{n-m-1}(f) \in T^{n-m-1}(f)$. La classe d'homologie $\bmod Y$ de $\gamma^{n-m-1}(f)$ ne dépend donc pas du choix du polyèdre $P^{n-m-1}$ et du prolongement (11.4).

En particulier, si $f$ est algébriquement prolongeable sur $\mathfrak{e}-Y$, on a $I^{n-m-1}(f)=0$, c. à d. $\gamma^{n-m-1}(f) \sim 0 \bmod Y$ dans $Q^{n}$. En vertu du lemme principal, il existe alors un sous-polyèdre $(n-m-2)$-dimensionnel $P^{n-m-2}$ de $Q^{n}$, tel que $\overline{P^{n-m-2}} \cdot X=0$ et que $f$ admet un prolongement $f\left(\mathcal{Q}-Y-P^{n-m-2}\right) C S^{m}$. D'autre part, pour tout cycle convergent $m$-dimensionnel $Z^{m}$ de $X$, la relation $Z^{m} \sim 0$ dans $\mathcal{e}-Y$ implique la relation $Z^{m} \sim 0$ dans ${ }^{e}-Y-P^{n-m-2}$. Un prolongement $f\left(\mathfrak{Q}-Y-P^{n-m-2}\right) \subset S^{m}$ n'est done possible que pour $f$ algébriquement prolongeahle sur $\mathfrak{Q}-Y$. Nous avons ainsi démontré le

Théorème III. Pour qu'une transformation continue $f(X) \subset S^{m}$ soit algébriquement prolongeable sur $\mathfrak{e}-Y$, il faut et il suffit qu'il existe un sous-polyeddre $(n-m-2)$-dimensionnel $P^{n-m-2}$ de $Q^{n}$, tel que $\overline{P^{n-m-2}} \cdot X=0$ et que $f$ admette un prolongement

$$
f\left(\mathfrak{a}-X-P^{n-m-2}\right) \subset S^{m}
$$

13. Theorème $\boldsymbol{I V}$. S'upposons que

$$
B^{i+1}(\mathfrak{e}-Y) \bmod X \text { coef. }\left(m^{l}\right)^{*}=0
$$

pour $i=m+1, m+2, \ldots, n-1$. Pour qu'une transformation continue $f(X) \subset S^{m}$ admette un prolongement $f\left(Q^{2}-Y\right) \subset S^{m}$, il faut et il suffit $q u$ 'elle soit algébriquement prolongeable sur $\mathrm{a}-X$. 
Théorème rVa. si l'on a (1.3.1), pour $i=m, m+1, \ldots, n-1$, toute transformation continue $f(X) \subset S^{m}$ admet un prolongement $f\left(\mathrm{a}^{\mathrm{D}}-\mathrm{Y}\right) \subset \mathrm{S}^{\mathrm{m}}$.

Le th. IV s'obtient du th. III et du th. I, appliqué successivement à $i=m+1, m+2, \ldots, n-1$.

14. Cas où a et $X$ sont des surfaces sphêriques. Admettons que $\mathfrak{Q}=S^{n}$ et que $X=S_{1}^{m}$ est une image homéomorphe de $S^{m}$. Soit $Z_{1}^{m}$ un cycle convergent de $S_{1}^{m}$ qui constitue une basse dans le groupe $B^{m}\left(S_{1}^{m}\right)$ coef. $\left(m^{m}\right)$ (c. à d. à coefficientis entiers).

A chaque transformation $\left.{ }^{30}\right) f\left(S_{1}^{m}\right) \subset S^{m}$ correspond un nombre entier $\mathrm{g}\left(f ; S_{1}^{m}\right)=\mathrm{g}\left(f ; Z_{1}^{m}\right)$ qui est le degré de cette transformation. Le th. IV peut être mis alors sous la forme qui suit:

Theorème $V$. Soit $S_{1}^{m} \subset S^{n}$ une image homéomorphe de $S^{m}$. Soit $Y \subset S^{n}-S_{1}^{m}$ un retracte absolu de voisinage, tel que

$$
B^{n-i-2}(Y) \text { coef. }\left(m^{i}\right)=0 \quad \text { pour } \quad i=m+1, m+2, \ldots, n-1 .
$$

Pour qu'une transformation continue $f\left(S_{1}^{m}\right) \subset S^{m}$ admette un prolongement $f\left(S^{n}-Y\right) \subset S^{m}$, il faut et il suffit qu'il existe un cycle convergent $Z_{2}^{n-m-1}$ de $\bar{Y}$ à coefficients entiers, tel que

$$
v\left(Z_{1}^{m} ; Z_{2}^{n-m-1}\right)=g\left(f ; S_{1}^{m}\right) .
$$

La condition est nécessaire. Admettons, en effet, l'existence du prolongement $f\left(S^{n}-\bar{Y}\right) C S^{m}$. A tout cycle convergent $Z^{m}$ de $S^{\prime \prime}-S_{1}^{m}$ à coefficients de $\left(m^{m}\right)^{*}$ correspond alors un élément $\mathrm{g}\left(f ; Z^{m}\right) \in\left(m^{m}\right)^{*}$, qui est son degré. On obtient ainsi une transformation homomorphe du groupe $B^{m}\left(S^{n}-Y\right)$ coef. $\left(m^{m}\right)^{*}$ en groupe $\left(m^{m}\right)^{*}$, c.̀̀d. on obtient le caractère du groupe $B^{m}\left(S^{n}-Y\right)$. Or, le groupe $B^{n-m-1}(Y)$ coef. $\left(m^{m}\right)$ étant orthogonal à $B^{m}\left(S^{n}-Y\right)$ coef. $\left.\left(m^{m}\right)^{*} 31\right)$, il existe un cycle convergent $Z_{2}^{n-m-1}$ de $Y$ à coefficients entiers, tel que l'on a

$$
v\left(Z^{m} ; Z_{2}^{n-m-1}\right)=g\left(f ; Z^{m}\right)
$$

pour tout cycle convergent $Z^{m}$ de $S^{n}-Y$ à coefficients de $\left(m m^{m}\right)^{*}$.

$\left.{ }^{30}\right)$ Dans tout l'article nous nous occupons seulement des transformations continues.

$\left.{ }^{31}\right)$ L. Pontrjagin, Ann, of Math. 35 (1934), p. 912.

Soit $\alpha \epsilon\left(m^{m}\right)^{*}$. Le cycle $\alpha Z_{1}^{m}$ est alors un cycle convergent à coefficients de $\left(m^{m}\right)^{*}$. Donc

d'où

$$
v\left(\alpha Z_{1}^{m} ; Z_{2}^{n-m-1}\right)=\mathrm{g}\left(f ; \alpha Z_{1}^{m}\right),
$$$$
\alpha v\left(Z_{1}^{m} ; Z_{2}^{n-m-1}\right)=\alpha g\left(f ; S_{1}^{m}\right) .
$$

Ceci étant vrai pour tout $a \epsilon\left(m^{m}\right)^{*}$, on en déduit (14.2).

La condition est suffisante. En vertu du (14.1), et du théorème de dualité ${ }^{31}$ ) on a en effet

$$
B^{i+1}\left(S^{n}-Y\right) \text { coef. }\left(m^{i}\right)^{*}=0 \quad \text { pour } \quad i=m+1, \ldots, n-1 .
$$

Soit $Z^{i+1}$ un cycle convergent $\bmod S_{1}^{m}$ de $S^{n}-Y$ à coefficients de $\left(m^{l}\right)^{*}(i=m+1, \ldots, n-1)$. $\partial Z^{i+1}$ étant in cycle convergent $i$-dimensionnel dis $S_{1}^{m}$ où $i>m$, il existe un cycle convergent ' $Z^{i+1}$ de $S^{n}-Y$ à coefficients de $\left(m^{i}\right)^{*}$, tel que ${ }^{\prime} Z^{i+1} \sim Z^{i+1}$ mod $S_{1}^{m}$ dans $S^{m}-Y$. En vertu de $(14.3)_{i}$ on a done $Z^{i+1} \sim 0 \bmod S_{1}^{m}$ dans $S^{m}-Y$. Il est ainsi établi que

$(14.4)_{i} \quad B^{i+1}\left(S^{n}-Y\right) \bmod S_{1}^{m}$ coef. $\left(m^{i}\right)^{*}=0 \quad$ pour $\quad i=m+1, \ldots, n-1$.

Soit $Z^{m+1}$ un cycle convergent mod $S_{1}^{m}$ de $S^{n}-Y$ à coefficients de $\left(m^{m}\right)^{*}$. Il existe alors un $\alpha \epsilon\left(m^{m}\right)^{*}$ tel que $\partial Z^{m+1} \sim \alpha Z_{1}^{m}$ dans $S_{1}^{m}$.

En vertiu de (1.4.2), on a alors

$$
\mathrm{g}\left(f ; \partial Z^{n+1}\right)=\alpha \mathrm{g}\left(f ; Z_{1}^{m l}\right)=\alpha v\left(Z_{1}^{m} ; Z_{2}^{n-m-1}\right)=v\left(\partial Z^{m+1} ; Z_{2}^{n-m-1}\right) .
$$

Il en résulte que $g\left(f: \partial Z^{m+1}\right)=v\left(\partial Z^{m+1} ; Z_{2}^{n-m-1}\right)=0$, puisque $\partial Z^{m+1} \sim 0$ dans $S^{n}-Y$.

La transformation $f$ étant ainsi algébriquement prolongeable sur $S^{n}-I$, il existe en vertu du th. IV un prolongement $f\left(S^{n}-Y\right) \subset S^{m}$.

Les conditions (14.1.), n'interviennent que dans la démonstration de la suffisance. Il est peut être intéressant de savoir si ces conditions sont essentielles pour le th. $V^{32}$ ).

Si l'on choisit comme $f\left(S_{1}^{m}\right) C S^{m}$ une transformation homéomorphe de degré 1 (done-ce qui revient au même - la transformation de $S_{1}^{m}$ en $S_{1}^{m}$ par l'identité), on a $g\left(f ; S_{1}^{m}\right)=1$ et le th. $\mathrm{V}$ donne une condition nécessaire et suffisante pour que $S_{1}^{m}$ soit un rétracte de $S^{n}-Y$.

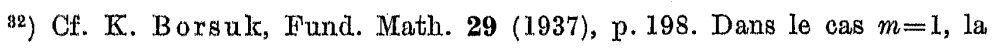
question a été résolue par l'affirmative (voir S. Eilenberg, Fund. Math. 28 (1937), p. 241). 
15. Cas où à, $X$ et $Y$ sont des surfaces sphêriques. Soient $S_{1}^{m} \subset S^{n}$ et $S_{2}^{n-m-1} \subset S^{n}-S_{1}^{m}$ des ensembles homéomorphes à $S^{m}$ et $S^{n-m-1}$ respectivement. Soient $Z_{1}^{m} \subset S_{1}^{m}$ et $Z_{2}^{n-m-1} C S_{2}^{n-m-1}$ deux cycles convergents à coefficients entiers qui forment respectivement des bases de $B^{m}\left(S_{1}^{m}\right)$ et de $B^{n-m-1}\left(S_{2}^{n-m-1}\right)$ à coefficients entiers. Posons

$$
\bar{v}\left(S_{1}^{m} ; S_{2}^{n-m-1}\right)=\left|v\left(Z_{1}^{m} ; Z_{2}^{n-m-1}\right)\right| .
$$

En posant $Y=S_{2}^{n-m-1}$, on constate que les conditions (14.1) sont satisfaites pour $i=m+1, \ldots, n-1$. Le th. $V$ implique done le

Théorème Va. Pour qu'une transformation continue $f\left(S_{1}^{m !}\right) \subset S^{m}$ admette un prolongement $f\left(S^{n}-S_{2}^{n-m-1}\right) \subset S^{m}$, il faut et il suffit que $\mathrm{g}\left(f ; S_{1}^{m}\right)$ soit un multiple de $\bar{v}\left(S_{1}^{m} ; S_{2}^{n-m-1}\right)$.

En choisissant comme $f$ la transformation de $S_{1}^{m}$ en $S_{1}^{m}$ par l'identité, on en déduit le

Théorème $\left.\boldsymbol{V b}{ }^{33}\right)$. Pour que $S_{1}^{m}$ soit un rétracte de $\mathrm{S}^{n}-S_{2}^{n-m-1}$, il faut et il suffit que

$$
\bar{v}\left(S_{1}^{m}, S_{2}^{n-m-1}\right)=1 .
$$

En particulier, si $S_{1}^{m}$ et un rétracte de $S^{n}-S_{2}^{n-m-1}$, alors $S_{2}^{n-m-1}$ et un rétracte de $S^{n}-S_{1}^{m}$.

16. Transformations algébriquement inessentielles. $\mathcal{P}$ étant un espace métrique quelconque, on dit que la transformation continue $f(\mathscr{P}) \subset S^{m}$ est algébriquement inessentielle, lorsque $\mathrm{g}\left(f, Z^{m}\right)=0$ pour tout cycle convergent $m$-dimensionnel $Z^{m}$ de $\mathscr{Z}$ à coefficients de $\left(m^{m}\right)^{*}$.

Soit $X \subset S^{n}$ un vrai sous-ensemble fermé de $S^{n}$. Soit $f(X) \subset S^{m}$. En vertu du th. II, il existe un polyèdre fini $(n-m-1)$-dimensionnel $P^{n-m-1} \subset S^{n}-X$ et un prolongement $f\left(S^{n}-P^{n-m-1}\right) \subset S^{m}$.

Théorème LIIa. Soit XCS ${ }^{n}$ un vrai sous-ensemble fermé de $S^{n}$. Pour qu'une transformation continue $f(X) \subset \mathcal{S}^{m}$ soit algebriquement inessentielle, il faut et il suffit qu'il existe un polyeddre fini $(n-m-2)$ dimensionnel $P^{n-m-2} C S^{n}-X$ et un protongement $f\left(S^{n}-P^{n-m-2}\right) \subset S^{n}$.

Ce th. résulte du th. III en y posant $\mathfrak{Q}=S^{n}$ et $Y=0$.

33) Pour lo cas $n=3, m=1$, voir $\mathrm{S}$. Eilenberg et K. Borsuk, Fund. Math. 26 (1936), p. 215.
Théorème $\left.V \boldsymbol{I}^{34}\right)$. Soit a un espace métrique compact de dimension finie et tel que

$$
B^{i}(囚) \text { coef. }\left(m^{i}\right)^{*}=0 \quad \text { pour } \quad i=m+1, m+2, \ldots
$$

Toute transformation continue $f(\curvearrowright) \subset S^{m}$ qui est algébriquement inessentielle est inessentielle.

En particulier, si on a aussi (16.1) $)_{m}$, alors toute transformation continue $f(2) \subset S^{m}$ est inessenticlle.

On peut admettre que $\mathfrak{e}^{e}=X \subset S^{n}$ où $\left.n>2 \mathrm{dim} \mathfrak{C}^{35}\right)$. Soit $X$ un ensemble composé d'un seul point de $S^{n}-X$. En vertu de (16.1), on a done:

$(1,6.2)_{l} \quad B^{i+1}\left(S^{n}-Y\right) \bmod X$ coef. $\left(m^{i}\right)^{*}=0$ pour $i=m+1, m+2, \ldots, n-1$. ot, d'après le th. TV, la fonction $f$ admet un prolongement $f\left(S^{n}-Y\right) C S^{m}$. Il en résulte que $f$ est une transformation inessentielle, puisque $s^{n}-Y$ est contractile dans soi.

\section{IIII}

17. Lemme $\left.{ }^{36}\right)$. Soit $A$ un sous-enscmble fermé d'un espace meitrique compact $\mathscr{Y}$. Soient $f_{1}(A) \subset S^{m}$ et $f_{2}(A) \subset S^{m}$ deux transformations ${ }^{30}$ ) homotopes. Pour tout prolongement $f_{1}(\mathscr{Q}) \subset S^{m}$, il existe un prolongement $f_{2}(\mathscr{\rho}) \subset S^{m}$ homotope à $f_{1}$ (sur of).

18. Lemme si deux transformations:

$$
f_{1}\left(X+Q^{n}-P^{k}\right) \subset S^{m}, \quad f_{2}\left(X+Q^{n}-P^{k}\right) \subset S^{m}
$$

sont homotopes, on a

$$
\gamma^{k}\left(f_{1}\right)=\gamma^{k}\left(f_{2}\right)
$$

On a, en effet, pour tout $a_{i}^{k}$ de $Q^{n}$

c. ̀̀ d. $\alpha_{i}\left(f_{1}\right)=\alpha_{i}\left(f_{2}\right)$

$$
\left[f_{1} \varphi_{i}^{n-k-1}\right]=\left[f_{2} \varphi_{i}^{n-k-1}\right]
$$

34) Ce thécorème renferme la solution affirmative (pour les espaces de di monsion finie) d'un problème posé par M. K. Borsuk, Fund. Math. 28 (1937), p. 210.

35) Voir W. Hurowicz, Sitzungsber. Preuss, Akad. 1933, p. 755

sa) K. Borsuk, Annales soc. Pol. de Math. 16 (1937), p. 218. Fundamenta Malhematicas. T.XXxI. 
19. Les ensembles $\boldsymbol{I}\left(\boldsymbol{a}_{i}^{k}\right)$. Soient $x_{1} \epsilon\left|\alpha_{i}^{k}\right|$ ot $x_{2} \epsilon\left|s_{i}^{n-k-1}\right|$. Thes points $x_{1}$ et $x_{2}$ appartiennent done ar même simplexe de $Q^{n}$ at lo segment rectiligne $x_{1} x_{2}$ est bien détermine. Lia somme de tous ces segments sera désignée par $I\left(\alpha_{i}^{h}\right)$. On vérifie sans peino quo doux segments ne peuvent avoir des points intórieurs commruns. Il en résulte immédiatement que

(19.1) $I\left(a_{i}^{k}\right)-\left|a_{i}^{k}\right|$ se laisse déformer dans lui-même en $\left|s_{i}^{n-k-1}\right|$.

L'ensemble $I\left(a_{i}^{k}\right)$ peut être aussi défini de la même manière en considérant les segments $x_{1} x_{2}$ pour $x_{1} \epsilon\left|\partial a_{i}^{k}\right|$ et $x_{2} \epsilon\left|b_{t}^{n-k}\right|$. On on déduit que

\section{(19.2) $I\left(a_{t}^{k}\right)-\left|\hat{c} a_{i}^{k}\right|$ se. laisse déformer dans lui-mìme an $\left|b_{i}^{n-k}\right|$.}

Soit $p_{i}$ le point dintersection de $a_{l}^{k}$ ot $b_{l}^{n-k}$. Lin remplagunt tout $x \in\left|b_{i}^{n-k}\right|$ par le centre *x du segment $x p_{\text {i }}$, on obtiont une es]lule ,concentrique" avec $b_{i}^{n-h}$; désignons-la par ${ }^{*} b_{i}^{n-k}$. Tal définition de ${ }^{*} I\left(a_{i}^{k}\right)$ est analogue à celle de $I\left(\alpha_{i}^{k}\right)$ : on n'a qu'à remplacer $b_{i}^{n-k} \operatorname{par}^{*} b_{i}^{n-k}$.

Soit $x_{1} x_{2}$ un segment tel que $x_{1} \epsilon\left|\hat{c} a_{i}^{k}\right|$ at $\left.x_{2} \epsilon\right|^{*} b_{i}^{n-\cdots k} \mid$. Pour tout $x \in x_{1} x_{2}$ où $x \neq x_{1}$, posons $r(x)=x_{2}$. On obtient ainsi une transformation continue

$$
\text { (19.3) }
$$

telle que:

$$
\begin{aligned}
& r(x)=x \quad \text { pour }\left.\quad x \in\right|^{*} b_{i}^{n-k} \mid \\
& \left.\left.r(x) \epsilon\right|^{*} s_{i}^{n-k-1} \mid \quad \text { pour } \quad x \in \operatorname{Srr}\left[{ }^{*} I\left(a_{l}^{k}\right)\right]-\left|2 a_{i}^{k}\right|^{37}\right) \text {. }
\end{aligned}
$$

Remarquons encore que la suite des ensembles $\left\{I\left(a_{l}^{h}\right)\right\}$, les $a_{l}^{k}$ parcourant tous les simplexes de $Q^{n}$, n'a aucun point d'accumulation dans $Q^{n}$.

20. Lemme. Etant donnée une transtormation

$$
f\left(X+Q^{n}-P^{k}\right) \subset S^{m}
$$

il existe un sous-polyedire $(z-1)$-dimensionnel $P^{k-1} \subset P^{k}$ de $Q^{n}$ et une transformation

$$
f_{1}\left(X+Q^{n}-\left|\gamma^{k}(f)\right|-P^{k-1}\right) \subset S^{m}
$$

qui est homotope à $f$ sur $X+Q^{n}-P^{k}$ et qui coüncide aveo $f$ sur $X$.

$\left.{ }^{37}\right) \operatorname{Fr}(A)$ désigne la frontière do l'ensemble $A$.
Soit $a_{1}^{k}, a_{2}^{k}, \ldots$ la suite de tous les $a_{i}^{k}$ de $P^{k}$ pour lesquels $\alpha_{i}(f)=0$, c. à d. pour lesquels $f$ transforme $\left|s_{i}^{n-k-1}\right|$ a'une façon inessentielle. En vertu de (19.1), $f$ est donc inessentielle sur $I\left(a_{i}^{k}\right)-\left|a_{i}^{k}\right|$ et, à plus forte raison, sur ${ }^{*} I\left(a_{i}^{k}\right)-\left|a_{i}^{k}\right|$. Posons

$$
V=\sum_{i=1}^{\infty} I\left(a_{i}^{k}\right)-\left|a_{i}^{k}\right|
$$

Les sommandes de $V$ étant disjoints, ouverts et fermés dans $V$, et la fonction $f$ étant inessentielle sur chacune d'elles, on conclut que $f$ est inessentielle sur $V$. Comme $V$ est un sous-ensemble fermé de $X+Q^{n}-P^{k}$ et disjoint de $X$, il existe en vertu de 17 une transformation $f_{2}\left(X+Q^{n}-P^{k}\right) \subset S^{m}$, homotope à $f$ et telle que

$$
f_{2}(x)=\left\{\begin{array}{ccc}
q & \text { pour } & x \in V \\
f(x) & \text { pour } & x \in X .
\end{array}\right.
$$

La transformation $f_{1}$ peut être définie comme suit:

$$
\begin{gathered}
P^{k-1}=\sum_{i=1}^{\infty}\left|\partial a_{i}^{k}\right|, \\
f_{1}(x)=\left\{\begin{array}{ccc}
f_{2}(x) & \text { pour } & x \in X+Q^{n}-P^{k} \\
q & \text { pour } & x \in V-P^{k-1}
\end{array}\right.
\end{gathered}
$$

21. Lemme. Soit $a^{k+1}$ un simplexe $(k+1)$-dimensionnel de $Q^{n}$ et $\beta \epsilon\left(m^{n-k-1}\right)$. Il existe une transformation

telle que:

$$
\begin{gathered}
g\left(X+Q^{n}-\left|\partial a^{k+1}\right|\right) \subset S^{m} \\
\gamma^{k}(g)=\beta \partial a^{k+1} \\
g(x)=q \quad \text { pour } \quad x \in X+Q^{n}-{ }^{*} I\left(a^{k+1}\right) .
\end{gathered}
$$

Soit $h\left(S^{n--k-1}\right) \subset S^{m}$ une transformation telle que $[h]=\beta$. Choisissons un $q^{\prime} \in S^{n-k-1}$ tel que $h\left(q^{\prime}\right)=q$ et une transformation

telle que

$$
\psi\left(\left|b^{n-k-1}\right|\right) \subset S^{n-k-1}
$$

$$
\psi(x)=q^{\prime} \quad \text { pour } \quad x \in\left|b^{n-k-1}\right|-\left.\right|^{*} b^{n-k-1} \mid
$$

et qui soit de degré 1 dans chaque point de $S^{n+k-1}$ distinct de $q^{\prime}$. Posons conformément à (21.2):

$$
g(x)=\left\{\begin{array}{cc}
q & \text { pour } x \in X+Q^{n}-{ }^{*} I\left(a^{k+1}\right), \\
h \psi r(x) & \text { pour } x \epsilon^{*} I\left(a^{k+1}\right)-\left|\partial a^{k+1}\right| .
\end{array}\right.
$$


Pour $x \in \operatorname{Fr}\left[{ }^{*} I\left(a^{k+1}\right)\right]-\left|\partial a^{k-1-1}\right|$, on a $\left.r(x) \epsilon\right|^{*} s^{n-k \mid} \mid$ d'après (19.5), (10) qui donne $\psi r(x)=q^{\prime}$ et $\operatorname{h} \psi r(x)=q$. Il en résulte que la transformation $g\left(X+Q^{n}-\left|a^{k+1}\right|\right) C S^{m i}$ est continue.

Soit

et posons

$$
\partial a^{k+1}=\sum \eta_{i} a_{i}^{k} \quad \text { où } \quad \eta_{i}= \pm 1
$$

$$
\psi_{i}(x)=\left\{\begin{array}{ccc}
\psi(x) & \text { pour } & x \in\left|b^{n-k-1}\right| \\
q^{\prime} & \text { pour } & x \in\left|s_{i}^{n-k-1}\right|-\left|b^{n-k-1}\right|
\end{array}\right.
$$

La transformation continue $\psi_{l}\left(\left|s_{i}^{n-k-1}\right|\right) \subset S^{n-k-1}$ ainsi définie est de degré $\eta_{i}$. La transformation $\psi_{i} \varphi_{i}^{n-k-1}\left(S^{n-k-1}\right) \subset S^{n-k-1-1}$ est donc aussi de degré $\eta_{i}$, d'où en vertu de (4.1)

$$
\left[h \psi_{i} \varphi_{l}^{n-k-1}\right]=\eta_{i} \beta
$$

et, comme $h w_{t}=\emptyset$ en vertil de (21.3), (19.4) et (21.4),

$$
\left[g \varphi_{i}^{n-k-1}\right]=\eta_{i} \beta \text {. }
$$

On a done $\alpha_{i}(g)=\eta_{i} \beta$ et $\gamma^{k}(g)=\sum \eta_{i} \beta a_{i}^{k}=\beta$ e $a^{k+1}$, (4) àd. (21.1).

22. Lemme. Etant donnes: une transformation

$$
f\left(X+Q^{n}-P^{h}\right) \subset S^{m}
$$

un simplexe $(k+1)$-dimensionnel $a^{k+1}$ de $Q$ et un element $\beta \in\left(m^{n-k-1}\right)$, il existe une transformation

$$
\begin{array}{lc} 
& j_{1}\left(X+Q^{n}-P^{k}-\left|\partial a^{k+1}\right|\right) \subset S^{m} \\
\text { telle que: } & \gamma^{k}\left(f_{1}\right)=\gamma^{k}(f)+\beta \partial a^{k+1}, \\
(22.1) & f_{1}(x)=f(x) \quad \text { pour } \quad x \in X+Q^{n}-P^{k}-I\left(a^{k+1}\right) .
\end{array}
$$

Ia transformation $f$ est inessentielle sur $\left|b^{n-k-1}\right| C Q-P^{k}$. En vertu de (19.2), elle est donc inessentielle sur $I\left(a^{k+1}\right)-\left|\partial a^{h-1+1}\right|$ et à plus forte raison sur ${ }^{*} I\left(a^{k+1}\right)-\left|\partial a^{k+1}\right|$. Les ensembles ${ }^{*} I\left(a^{k+1}\right)-\left|\partial a^{k-1}\right|$ et $X+Q^{n}-P^{k}-I\left(a^{k+1}\right)+\operatorname{Fr}\left[I\left(a^{k+1}\right)\right]-\left|c a^{k+1}\right|$ étant disjoints et fermés dans $X+Q^{n}-P^{k}-\left|\partial a^{k+1}\right|$, il existe en vertu de $\mathbf{1 7}$ une transformation

$$
f_{2}\left(X+Q^{n}-P^{k}-\left|\partial a^{k+1}\right|\right) \subset S^{m}
$$

homotope à $f$ et telle que:

$\begin{array}{llll}(22.3) & f_{2}(x)=f(x) & \text { pour } & x \in X+Q^{n}-P^{k}-I\left(a^{k+1}\right) \\ (22.4) & f_{2}(x)=q & \text { pour } & x \in \epsilon^{*} I\left(a^{k+1}\right)-\left|\partial a^{k+1}\right|\end{array}$

En vertu de 18, on a donc

Posons

(22.6) $\quad f_{1}(x)=\left\{\begin{array}{lll}f_{2}(x) & \text { pour } & x \in X+Q^{n}-P^{k}-{ }^{*} I\left(a^{k+1}\right), \\ g(x) & \text { pour } & x \epsilon^{*} I\left(a^{k+1}\right)-\mid \partial a^{k+1} .\end{array}\right.$

où $g$ désigne la fonction donnée par 21. Il résulte de (21.2), (22.4) et (22.3) que la transformation $f_{1}\left(X+Q^{n}-P^{k}-\hat{c} a^{k+1} \mid\right) C S^{m}$ est continue et satisfait à (22.2).

Reste à établir (22.1). En vertu de (22.1) et de (22.5), il suffit à ce but de démontrer que

$$
\gamma^{k}\left(f_{1}\right)=\gamma^{k}\left(f_{2}\right)+\gamma^{k}(g)
$$

Soit $a_{i}^{k}$ un simplexe $k$-dimensionnel de $Q$. Deux cas sont à distinguer:

$1^{0}\left|a_{i}^{k}\right|$ non $C\left|\partial a^{k+1}\right|$. On a alors $\left|s_{i}^{n-k-1}\right| \subset Q^{n}-{ }^{*} I\left(a^{k+1}\right)$. En vertu de (22.6) et de (21.2), on a dono:

$$
f_{1}(x)=f_{2}(x) \quad \text { et } \quad g(x)=q \text { pour } \quad x \in\left|s_{i}^{n-k-1}\right|,
$$

ce qui implique que:

$$
\alpha_{i}\left(f_{1}\right)=\alpha_{i}\left(f_{2}\right), \quad \alpha_{i}(g)=0
$$

et, par conséquent, que $a_{i}\left(f_{1}\right)=\alpha_{i}\left(f_{2}\right)+a_{i}(g)$.

$2^{0}\left|a_{i}^{k}\right| C\left|\partial a^{h+1}\right|$. On a alors $\left|b^{n-k-1}\right| C\left|s_{i}^{n-k-1}\right|$, donc aussi

$$
\left|b^{n-k-1}\right| \subset\left|s_{i}^{n-k-1}\right| \text {. }
$$

Choisissons la transformation continue de degré 1

de façon que l'on ait:

$$
\varphi_{i}^{n-k-1}\left(S^{n-k-1}\right) \subset\left|s_{i}^{n-k-1}\right|
$$

$$
\begin{gathered}
\left.\varphi_{i}^{n-k-1}\left(S_{+}^{n-k-1}\right) \subset\right|^{*} b^{n-k-1} \mid, \\
\varphi_{i}^{n-k-1}\left(S^{n-k-1}-S_{+}^{n-k-1}\right) \subset\left|s_{i}^{n-k-1}\right|-\left.\right|^{*} b^{n-k-1} \mid,
\end{gathered}
$$

où $S_{+}^{n-k-1}$ est une hémisphère de $S^{n-k-1}$. Comme on a en vertu de (22.6)

$$
f_{1}(x)=\left\{\begin{array}{lll}
g(x) & \text { pour } & \left.x \in\right|^{*} b^{n-k-1} \mid \\
f_{2}(x) & \text { pour } & x \in\left|s_{i}^{n-k-1}\right|-\left.\right|^{*} b^{n-k-1} \mid,
\end{array}\right.
$$


il vient conformément à la définition de l'addition dans lo groupe $\left(m^{n-k-1}\right)$ (voir 4)

$$
\left[f_{1} \varphi_{i}^{n-k-1}\right]=\left[f_{2} p_{l}^{n-k-1}\right]+\left[y \phi_{l}^{n-k-1}\right],
$$

c. ̀̀ d. $\alpha_{i}\left(f_{1}\right)=\alpha_{i}\left(f_{2}\right)+\alpha_{i}(g)$.

23. Lemme. Etant données: une transformation

$$
f\left(X+Q^{n}-P^{k}\right) \subset S^{m}
$$

et une châ̂ne infinie $(k+1)$-dimensionnelle $\Lambda^{k+1} \bmod X$ à coefficients de $\left(m^{n-k-1}\right)$, il existe un sous-polyedre $P_{1}^{k}$ de $Q^{n}$ tel que $\left.P_{1}^{k} \cdot X=0{ }^{38}\right)$ et une transformation

telle que:

$$
f\left(X+Q^{n}-P_{1}^{h}\right) \subset S^{m}
$$

$$
\begin{gathered}
\gamma^{k}\left(f_{1}\right)=\gamma^{k}(f)+\partial \Lambda^{k+1}, \\
f_{1}(x) \Rightarrow f(x) \quad \text { pour } \quad x \in X^{x} .
\end{gathered}
$$

Soit $a_{1}^{k+1}, a_{2}^{k+1}, \ldots$ la suite de tous les simplexes $(k+1)$-dimensionnels de $\left|\Lambda^{k+1}\right|$. On a donc

$$
\Lambda^{k+1}=\sum_{i=1}^{\infty} \beta_{t} a_{t}^{k+1} \text { où } \beta_{t} \in\left(m^{n-k-1}\right), \beta_{i} \neq 0 .
$$

Posons

$$
P_{1}^{k}=P^{k}+\sum_{i=1}^{\infty}\left|\partial a_{i}^{k+1}\right|
$$

On a $P_{1}^{k} \subset P^{k}+\left|\Lambda^{k+1}\right|$, d'où $\overline{P_{1}^{k}} \cdot X=0$, puisque $\Lambda^{k+1}$ este une chaîne $\bmod Y$.

Soient $f^{(0)}=f$ et $f^{(i)}$ la fonction qu'on obtient en appliquant $\mathbf{2 2}$ à $f^{(i-1)}, a_{i}^{k+1}$ et $\beta_{i}$. Conformément à $\mathbf{2 2}$, on a done:

$$
\begin{gathered}
f^{(i)}\left(X+Q^{n}-P_{1}^{k}\right) \subset S^{m}, \\
\gamma^{k}\left(f^{(l)}\right)=\gamma^{k}(f)+\partial\left(\sum_{\gamma=1}^{L} \beta_{j} a_{j}^{k+1}\right), \\
f^{(i)}(x)=f^{(l-1)}(x) \text { pour } x \in X+Q^{n}-P_{1}^{k}-I\left(a_{i}^{k+1}\right) .
\end{gathered}
$$

Or, $\Lambda^{k+1}$ étant une chaîne mod $X$, tous les points d'accumumlation de la suite $\left\{I\left(a_{l}^{k+1}\right)\right\}$ appartiennent à $Y$. Pour tout ensemble

\footnotetext{
88) On a ṇềme $P_{1}^{k} \subset P^{k}+\left|\Delta^{k+1-1}\right|$,
}

compact $Z C X+Q^{n}-P_{1}^{k}$, il existe done un $i_{0}$ tel que $Z \cdot I\left(a_{i}^{k+1}\right)=0$ pour $i \geqslant i_{0}$. Par conséquent

$$
f^{(i)}(x)=f^{\left(t_{0}\right)}(x) \quad \text { pour } x \in Z \quad \text { et } i>i_{0}
$$

et il ne reste qu'a poser

$$
f_{1}(x)=\lim _{i \rightarrow \infty} f^{(i)}(x) \quad \text { pour } \quad x \in X+Q-P_{1}^{k} .
$$

24. Lemme. Etant donnés: une division simpliciale $Q^{n}$ de $S^{n}$, un sous-polyèdre 0-dimensionnel $P^{0}$ de $Q^{n}$ et une transformation

$$
f\left(S^{\prime \prime}-P^{0}\right) \subset S^{m}
$$

la somme des coefficients de $\gamma^{0}(f)$ est égale à 0 .

Soient $a_{1}^{0}, a_{2}^{0}, \ldots, a_{r}^{0}$ les simplexes de $P^{0}$. On a

$$
\gamma^{0}(f)=\sum_{i=1}^{r} \alpha_{i}(f) a_{i}^{0}
$$

Posons

$$
\gamma_{1}^{0}=\sum_{i=1}^{r} \alpha_{i}(f) a_{1}^{0}
$$

Comme la somme des coefficients de $\gamma^{0}(f)$ est égale à celle de $\gamma_{1}^{0}$, il existe une chaine 1-dimensionnelle $\Lambda^{1}$ de $Q^{n}$ à coefficients de $\left(m^{n-1}\right)$, telle que

$$
\gamma_{1}^{0}=\gamma^{0}(f)+\Lambda^{1}
$$

En vertu de $\mathbf{2 3}$, il existe un sous-polyèdre 0-dimensionnel $P_{1}^{0}$ de $Q^{n}$ et une transiormation $f_{1}\left(S^{n}-P_{1}^{0}\right) \subset S^{m}$ telle que $\gamma^{0}\left(f_{1}\right)=\gamma_{1}^{0}$. Comme $\left|\gamma^{0}\left(f_{1}\right)\right|=\left|a_{1}^{0}\right|$, on peut admettre en vertu de $\mathbf{2 0}$ que $P_{1}^{0}$ contient seulement le sommet $a_{1}^{0}$. Mais alors $f_{1}$ est une transformation inessentielle, puisque $S^{n}-\left|a_{1}^{0}\right|$ est contractile dans soi. Par conséquent $\gamma_{1}^{0}=\gamma^{0}\left(f_{1}\right)=0$ et, d'après $(24.1), \sum_{i=1}^{r} \alpha_{i}(f)=0$, c. q. t. d.

25. Démonstration du lemme principal. Soit $a^{k-1}$ un simplexe $(k-1)$-dimensionnel arbitraire de $Q^{n}$. Soient $a_{1}^{k}, a_{2}^{k}, \ldots, a_{r}^{k}$ tous les simplexes $k$-dimensionnels de $Q^{n}$ contenant $a^{k-1}$ comme face. Soit $\eta_{i}$ le coefficient de $a^{k-1}$ dans $\partial a_{i}^{k}$. On a alors

$$
s^{n-k}=\sum_{i=1}^{r} \eta_{i} b_{i}^{n-k} .
$$


Désignons par $p_{i}$ le point d'intersection de $a_{i}^{h}$ st $b_{i}^{n-k}$ (c. id d. le centre de gravité de $a_{i}^{h}$ ) et par $\hat{p}^{0}$ le polyèdre ()-dimensionnel composé de points $p_{1}, p_{2}, \ldots, p_{r}$. On a alors

$$
\left|s^{n-k}\right|-\hat{P}^{0} \subset Q-P^{k} \text {. }
$$

Le polyèdre $\left|s^{n-k}\right|$ est homéomorphe à $S^{n-k}$ et la première subdivision barycentrique de $Q^{n}$ fournit une division simpliciale $\hat{Q}^{n-k}$ de $\left|s^{n-k}\right|$. Le point $p_{i}$ devient alors un sommet $\hat{a}_{i}^{0}$ et $\hat{p}^{0}$ devient un sous-polyèdre de $\hat{Q}^{n-h}$.

Toute transformation $f\left(X+Q^{n}-P^{h}\right) C S^{m}$ induit an vertu de (25.2) une transformation $f\left(\hat{Q}^{n-k}-\hat{P}^{0}\right) \subset S^{m}$; celle-ci nous fournit la chaîne $\gamma^{0}(f)$ et les éléments $\hat{a}_{i}(f) \epsilon\left(m^{n-h-1}\right)$ pour $i=1,2, \ldots, r$.

D'après $\mathbf{2 4}$, on a done

$$
\sum_{l=1}^{r} \hat{\alpha}_{l}(f)=0
$$

La cellule $\hat{b}_{i}^{n-k}$, duale $\grave{a} \hat{a}_{l}^{0}$ dans $\hat{Q}^{n-k}$, est contenuo dans la celIule $b_{i}^{n-k}$ et, en verta de (25.1), elle est orientíe comme $\eta_{l} b_{l}^{n-h}$. Par conséquent $\hat{\alpha}_{i}(f)=\eta_{t} \alpha_{i}(f)$ et d'après (25.3) $\sum_{i=1}^{r} \eta_{i} \alpha_{i}(f)=0$.

Comme c'était le coefficient de $a^{k-1}$ dans la chaine infinio $\partial\left[\gamma^{k}(f)\right]$, la chaîne $\gamma^{k}(f)$ est un cycle infini. Il résulte de $(5.5)$ qu'elle est un cycle inlini $\bmod Y$.

Supposons maintenant que $\gamma^{k}(f) \sim 0 \bmod X$. Soit dono $\Lambda^{k+1}$ la chaîne infinie $(k+1)$-dimensionnelle mod $X$ is coeflicientis de $\left(m^{n-k-1}\right)$,
telle que

$$
\gamma^{k}(f)+\partial \Lambda^{k+1}=0
$$

En vertu de 23, il existe un sous-polyèdre $k$-dimensionnel $P_{1}^{k}$ de $Q$ tel que $\overline{P_{1}^{k}} \cdot X=0$, et une transformation $f_{1}\left(X+Q-P_{1}^{k}\right) \subset S^{m}$ telle que $\gamma^{k}\left(f_{1}\right)=0$ et $f_{1}(x)=f(x)$ pour $x \in X$. Comme $\cdot\left|\gamma^{k}\left(f_{1}\right)\right|=0$, on trouve, en appliquant $\mathbf{2 0}$, un polyèdre $(k-1)$-dimensionnel
$P^{k-1} \subset P_{1}^{k}$ et une transformation

$$
f^{*}\left(X+Q-P^{k-1}\right) \subset S^{m}
$$

qui coïncide sur $X$ avec $f_{1}$, done aussi avee $f$.

\section{On the equivalence of any set of first category to a set of measure zero.

\author{
By
} \\ J. C. Oxtoby and S. M. Ulam (Cambridge, U. S. A.).}

In $n$-dimensional euclidean space $R^{(n)}$ it is an easy matter to define sets of first category which have positive measure, and even, ones whose complements are of measure zero. For instance, the complement of the intersection of any sequence of dense open sets whose measures tend to zero defines such a set. Nevertheless one may ask whether such a set is equivalent to a set of measure zero under the group of homeomorphisms of the space onto itself. In other words, does there exist an automorphism (that is, a homeomorphism of the space onto itself) which carries the given set into one of measure zero. The object of the present note is to show that such a transformation always exists, indeed that the automorphisms which carry a given set of first category into one of measure zero form a residual set in the space of antomorphisms, provided the latter is suitably metrised.

To begin with, consider the unit cube $g^{(n)}$ in $n$-dimensional euclidean space, $(n \geqslant 1)$. Let $[H]$ denote the space of all automorphisms of $g^{(n)}$ metrise by the formula $\left.{ }^{1}\right)$

$$
\varrho(g, h)=\max _{x \in \mathcal{J}^{\mathcal{J}}(n)}\left(|g x-h x|,\left|g^{-1} x-h^{-1} x\right|\right),
$$

where $|x-y|$ denotes the euclidean distance between $x$ and $y$. The space $[H]$ is complete and the group operations are continuous in the metric, so that it forms a metric group.

1) This metric is equivalent to the usual one. See S. Banach, Théorie des opérations linéaires, Monografie Matematyczne 1, Warszawa 1932, p. 229. 